\title{
PROBLEMAS CON EL LENGUAJE CIENTÍFICO EN LA ESCUELA. UN ANÁLISIS DESDE LA OBSERVACIÓN DE CLASES DE CIENCIAS NATURALES
}

\author{
GALAGOVSKY, L.R., BONÁN, L. y ADÚRIZ BRAVO, A. \\ Centro de Formación e Investigación en Enseñanza de las Ciencias. \\ Facultad de Ciencias Exactas y Naturales, Universidad de Buenos Aires. \\ Ciudad Universitaria, Pabellón II. 1428 Buenos Aires. Argentina.
}

\section{SUMMARY}

The present report describes examples and reaches conclusions concerning failures in class communication between science teachers and their students. A general situation characterised as «empty discourse» could be observed through analysing classes recorded by students belonging to last year in Science Teaching degrees. We inspect some implications of our observations for pre-service teachers' training.

\section{INTRODUCCIÓN}

En el marco de la asignatura Didáctica Especial y Práctica de la Enseñanza, última materia de los profesorados en física, química y biología de la Facultad de Ciencias Exactas y Naturales de la Universidad de Buenos Aires, se llevaron a cabo durante 1995 observaciones de clases en distintas instituciones de nivel secundario de la ciudad de Buenos Aires y su conurbano. El objeto de tales observaciones fue el de desarrollar diferentes planos de análisis para evaluar la eficiencia de los procesos comunicativos que ocurren en las clases de ciencias naturales, y tomar esos planos como indicadores que permitieran repensar el futuro rol docente de los observadores, con implicancias para su formación en el profesorado.

Luego de concretar las observaciones en un número relativamente grande de escuelas con diferentes perfiles, quedaron al descubierto problemas sistemáticos de comunicación en el aula. El análisis de los registros de dichas observaciones nos permitió inferir la existencia de mecanismos generales de vaciamiento discursivo en el aula. Entendemos por vaciamiento discursivo la desnaturalización de la función del lenguaje como sustento de los contenidos disciplinares específicos, sin perjuicio de otras funciones explícitas u ocultas. Entendemos, también, que se trata de un vaciamiento y no de un malentendido, porque los actores involucrados -docentes y alumnos observados-no parecían ser concientes de dicha pérdida de sentido, sino que más bien la reforzaban desde actitudes que catalogamos como:

- un hacer como si los alumnos entendieran, aun cuando las evidencias de errores conceptuales graves en los exámenes desmienten tal comprensión.

- un diálogo de sordos, donde el observador externo advierte claramente que docente y alumnos enmarcan 
sus discursos en referenciales semánticos diferentes y, consecuentemente, no se produce interacción genuina de significados.

- una negociación de poder, por la cual los docentes transfieren el problema de la incomunicación a los alumnos o a factores externos a la relación didáctica.

\section{LOS OBJETIVOS}

Los objetivos que guiaron la elaboración del presente trabajo pueden resumirse en tres instancias:

1. Establecer un espacio en la formación de los practicantes -futuros profesionales de la enseñanza-para el análisis crítico de prácticas pedagógicas y la identificación de mecanismos cotidianos que obstaculizan la comunicación en el aula.

2. Esbozar un marco teórico que dé soporte a la reflexión acerca de las situaciones observadas.

3. Analizar y ejemplificar, desde el marco teórico, distintos casos que revelan cómo se cometen acciones sistemáticas vaciadoras del discurso escolar.

\section{PRESUPUESTOS METODOLÓGICOS ACORDADOS}

El grupo de practicantes que asumió la tarea de analizar los registros de observación de clase consensuó una serie de supuestos previos para facilitar la tarea de análisis posterior:

1. El observador no interfiere de forma significativa en el desarrollo de la clase.

2. El docente observado ha aceptado tal situación en los términos en los que se concibe la observación de clases dentro del marco de la formación de nuevos profesores (no como una evaluación de su desempeño frente a los alumnos).

3. El profesor observado asume frente al observador un perfil de profesional docente con amplia experiencia y despliega parte de su arsenal de estrategias didácticas durante su desenvolvimiento al frente del curso.

4. Nuestra observación no intenta evaluar el conocimiento específico real de la asignatura que tienen los docentes observados.

\section{METODOLOGÍA DE TRABAJO}

\section{La selección de las escuelas}

Las instituciones disponibles para realizar las observaciones cubrieron un amplio espectro de modalidades de educación, formas de organización administrativa, niveles socioeconómicos reclutados en la matrícula, y confi- guraciones pedagógicas y curriculares. Se observó a veinticuatro docentes en once establecimientos. Los nueve observadores presenciaron y registraron un total de noventa y seis horas de clase, correspondientes a asignaturas con diferentes orientaciones disciplinares dentro de las ciencias naturales, de cursos de escuela secundaria (comprendida entre los 13 y 18 años).

\section{El método de observación}

Ante la imposibilidad de aislar y medir todas las variables presentes en una práctica docente real, se optó por el método de observación natural (Easley, 1982), no restringido por pautas escritas de ninguna índole. Se puso especial énfasis en el registro cuidadoso de las interacciones lingüísticas entre docentes y alumnos.

En reuniones preliminares se decidió no utilizar durante las observaciones ninguno de los materiales escritos (grillas, planillas) previamente elaborados que habíamos recolectado de diversas fuentes, en razón de su insuficiencia teórica y de que generalmente éstos enfocaban la cuestión sobre la «calidad académica» del desempeño del docente. Consecuentemente, los observadores (del tipo no participante), se abocaron al registro magnetofónico y taquigráfico de las interacciones lingüísticas que tenían lugar durante el transcurso de las clases. A ello se sumó un registro libre de impresiones y comentarios generales sobre los modelos pedagógicos, vinculares y didácticos observados. Los alumnos de la asignatura didáctica especial debieron presentar el análisis de estos registros como parte de las tareas a cumplir para obtener la aprobación. Son estos registros de interacciones lingüísticas los que constituyen el material documental a partir del cual se realizó el análisis del presente artículo.

\section{Organización del marco teórico}

Los registros de observación

Las observaciones se realizaron durante un período de dos meses. Reuniones semanales del grupo de observadores y docentes de la cátedra permitieron encontrar pautas comunes de análisis y encauzar el enfoque de las observaciones. Las pautas de análisis consistieron en enfocar el trabajo sobre segmentos llamativos de la clase intentando interpretar las diferentes construcciones personales de significados derivadas de un mismo hecho comunicativo. Estas pautas se transformaron en categorías al tratar de comprender y sistematizar los obstáculos encontrados en la comunciación didáctica. Por último, de los registros de observación obtenidos, fueron extraídos ejemplos representativos y organizados en coherencia con dichas categorías de análisis.

\section{Análisis de casos}

Aunque los resultados no fueron analizados estadísticamente, encontramos valioso el aporte de una metodología de análisis de casos (Ander-Egg, 1980) sobre algu- 
nos ejemplos en los que pueden rastrearse tendencias comunes y situaciones extrapolables. El estudio del material disponible condujo a observaciones que ayudan a repensar las propias prácticas docentes de los futuros egresados del profesorado a la luz de conceptualizar al docente como comunicador (Contreras, 1990).

\section{MARCO TEÓRICO}

Para abordar la tarea de clasificar los informes de observación y de presentar algunos resultados en forma coherente, fue necesario recortar el proceso multivariado de enseñanza-aprendizaje. El plano elegido para el recorte es el lingüístico, en el cual nos detuvimos para poner especial énfasis en las fracturas del proceso de enseñanza-aprendizaje como comunicación (Contreras, 1990; Pozo et al., 1991).

De las observaciones realizadas emerge la existencia de «acuerdos tácitos» (negociaciones implícitas) entre los actores de los distintos niveles de la institución escolar (directivos, docentes y alumnos), que determinan en gran medida la dinámica institucional. Estos acuerdos se ponen de manifiesto en el modo particular en que se emplea el lenguaje natural como interfase entre los componentes del triángulo didáctico (docente, alumnos, contenidos).

\section{El lenguaje como mediador}

Docente, alumnos y contenidos se relacionan en el aula a través de un riquísimo conjunto de prácticas no lingüísticas, pero es sin duda el lenguaje natural el medio a través del cual se produce la parte más significativa del proceso de enseñanza-aprendizaje. El lenguaje es el mediador de las articulaciones cognitivas entre el docente y los alumnos, en una postura que considera al proceso como una negociación de significados (Contreras, 1990; Edwards y Mercer, 1988; Gimeno Sacristán y Pérez Gómez, 1985). El lenguaje, a su vez, es vehículo de construcción de significaciones comunicables y compartibles sólo cuando el aprendizaje mismo está cargado de significatividad.

Podemos definir el lenguaje en este contexto como el tránsito en una superficie, que representa la negociación de significados, siendo la palabra el símbolo relacionado con esos significados. Un concepto formulado en una palabra representa cierto grado de generalización, y la relación entre conceptos es una relación de generalidad (Vygotsky, 1993).

\section{El lenguaje y los desencuentros}

El lenguaje natural funciona normalmente como mediador de las prácticas entre docentes y alumnos, y como sustento y formato de los contenidos específicos. Sin embargo, se produce muy frecuentemente un vaciamiento del discurso escolar; es decir, hay una ruptura, tergi- versación de los mensajes puestos en juego en la clase independientemente de qué roles asuman docente o alumnos.

Postulamos como organizador de nuestro análisis de las observaciones que el lenguaje falla en sus funciones en, por lo menos, tres categorías diferentes:

\section{Categoría $a$}

- Cuando el lenguaje disciplinar -de uso especial y restringido, altamente específico, denotativo y unívococontrasta con la vaguedad, polisemia y riqueza connotativa del lenguaje del sentido común que maneja el alumno (Lahore, 1993), sin que éste sea conciente del salto desde un contexto semántico a otro (Galagovsky, 1993 y 1996).

\section{Categoría $b$}

- Cuando los contenidos tienen un nivel de abstracción tan alto que su manipulación lingüística se torna difícil para el alumno (Borsese, 1994).

\section{Categoría $c$}

- Cuando la transposición didáctica (Chevallard, 1997) está distorsionada en el docente o en el material de trabajo, y es tan radical que se transforma en sustitución patológica; esto es, el contenido disciplinar está desnaturalizado (Frigerio, 1991).

Es de notar que los tres mecanismos seleccionados conducen a un resultado prácticamente idéntico: el $v a$ ciamiento en la significación de los contenidos que se intenta enseñar. Los términos científicos quedan como formas lógicas sin referencia a imágenes mentales, o refiriéndose a imágenes desvirtuadas (Merzyn, 1987).

Estos mecanismos determinan una fractura de la comunicación didáctica y del lenguaje como interfase, que conduce a un simulacro de negociación de significados, donde docentes y alumnos, en una especie de «diálogo de sordos», manipulan el lenguaje de forma tal de hacer creer al otro que hay compromiso con la tarea, cuando en realidad se ha producido un vaciamiento conceptual. Esta situación ritualizada de hacer como que se enseña y hacer como que se aprende en el aula (llamada por Cañal y Porlán [1987] el modelo de enseñanza-ficción) suele presentarse clara para el observador externo, pero en general parece no ser percibida por los actores (por lo menos, en el caso de los docentes) la mayor parte del tiempo.

\section{ALGUNOS EJEMPLOS CONCRETOS}

Una vez elegidas las variables que decidimos estudiar, procedimos a extraer de los noventa y seis registros toda la información que consideramos relevante y a organizarla de acuerdo a nuestro marco teórico. Presentamos a continuación algunos ejemplos que ilustran nuestro análisis. Las citas son textuales. Los diálogos selecciona- 
dos, o algunas frases específicas extraídas de ellos, se presentarán con un breve contexto y discusión.

\section{¿Qué entienden los alumnos cuando... ?}

Ejemplo 1

El docente dice:

Cuando dos cuerpos están en contacto, el cuerpo más caliente le transmite calor al cuerpo más frío.

En este ejemplo puede verse cómo el docente citado no tiene en cuenta la diferencia entre recibir un término desde su formato lingüístico y comprenderlo desde su contenido conceptual (Galagovsky, 1996), ignorando además la dificultad adicional que supone la fuerte carga de contenido no científico presente en los términos calor y caliente en el lenguaje del alumno (Erickson y Tiberghien, 1992). Una oración más rigurosa desde lo científico debería omitir el término caliente y hacer referencia a la temperatura. La oración es absolutamente comprensible para los alumnos, pero encierra fallas semánticas desde el lenguaje científico (categoría $a$ ). Al haber sido dicha por el docente, no suscita en los alumnos la menor duda acerca de su validez.

\section{Ejemplo 2}

En la clase de física, durante el tema de las incertezas experimentales, una alumna acota:

-De la exactitud.

Y la profesora corrige.

-De la «pre-ci-sión».

El aporte del término científico correcto «nominaliza» la situación (Bonán y Adúriz Bravo, 1995); pero, no mediando mayor explicación, ni explorando los significados otorgados a ambas palabras, se deja de lado el problema de la representación mental que tiene la alumna del concepto de exactitud/precisión. Éste es otro ejemplo de la categoría $b$.

\section{Ejemplo 3}

Alumno: - ¿Cuando dice vacío, se refiere al aire o al vacío-vacío?

Profesor:-Al vacío-vacío-vacío, pero es más o menos lo mismo.

Aquí se ha omitido completamente el problema de las representaciones alternativas (precientíficas) que el alumno vuelca en el término vacío (Séré, 1989).

Tanto en el ejemplo 2 como en el 3, la negociación de los significados se ha llevado al plano exclusivamente lingüístico y el docente no pone cuidado en investigar lo que los alumnos sacan en claro de su discurso. La polisemia del lenguaje cotidiano se contrapone a la significación unívoca del lenguaje científico, dificultando la comunicación por la inexistencia de negociación y por la ignorancia de estas mediaciones (Entel, 1988).

\section{Un diálogo de sordos}

En muchos casos observados, notamos por parte del docente un desconocimiento de la dificultad que introduce el alto componente de formalización de las disciplinas:

\section{Ejemplo 4}

Un profesor de física dice:

-En un segundo, la Tierra recorre la distancia desde Buenos Aires hasta San Fernando.

Una alumna manifiesta una incoherencia semántica:

-Pero si San Fernando está en la Tierra...

El profesor cierra toda discusión con un:

-No, hija, no entendiste nada.

Y continúa su explicación.

En este ejemplo, la alumna no puede abstraer la idea de la magnitud de la separación entre dos puntos sobre la superficie de la Tierra como patrón de medida para el propio desplazamiento angular del planeta (categoría $b$ ). El docente no parece darse cuenta del problema generado por el salto contextual.

En este caso, como en otros clasificados en la misma categoría, los docentes tienden a desconocer al lenguaje como mediador (Borsese, 1994), al no tomar conciencia de que la palabra representa un grado de generalización creciente que se corresponde con distintos niveles de evolución de los significados (Vygotsky, 1993). En estas situaciones, si no se explicita la (nueva) denotación de los términos y no se toma en cuenta la posible connotación, el docente no reflexiona acerca del fenómeno de transposición didáctica presente en su propio discurso. Da un significado único, no negociable, a sus palabras, que deben ser asumidas como «la verdad», ignorando significaciones alternativas posibles.

\section{Ejemplo 5}

Una profesora de biología indica:

- La finalidad del ciclo de Krebs es la de liberar dióxido de carbono y obtener NADH.

Algunos alumnos se manifiestan gestualmente, siendo esto indicio claro - para el observador- de que no hallan contexto para interpretar la sentencia de la profesora, quien ha utilizado términos científicos que no encuentran significante apropiado en la estructura cognitiva de los alumnos (Ausubel et al., 1983). Cabe destacar además el uso del término finalidad, con connotaciones más 
teleológicas que funcionales (Gaeta et al., 1996), lo que sitúa a la frase peligrosamente cerca de una visión «vitalista».

\section{Ejemplo 6}

Durante una clase de química, la profesora hace referencia constante a los conceptos de uniones químicas, niveles energéticos y electronegatividad. Los alumnos manifiestan explícitamente no entender el significado y la aplicación de estos conceptos, aunque operan con ellos siguiendo el discurso de la profesora, que no se detiene ante las dudas.

\section{Ejemplo 7}

En otra clase de química, la profesora interroga sobre hidrácidos (tema de la clase anterior) y señala a una alumna para que responda. Ésta lee mecánicamente de la carpeta preguntando dónde debe detenerse.

\section{Hacer «como que se enseña»}

En los siguientes ejemplos, los profesores utilizan términos del lenguaje vulgar con la intención de que los alumnos entiendan. Sin embargo, no hay momentos en la clase durante los cuales se hace explícita esta sustitución; la información real que se transmite está desplazada desde su denotación científica original (categoría $c$ ). Nuevamente llegamos a un vaciamiento, esta vez uniendo conceptos científicos a imágenes mentales erróneas y concretas. Y, al producirse la fractura comunicativa, los actores se refugian en una representación estereotipada de roles tradicionales en la cual cada uno dice lo que el otro espera escuchar (Cañal y Porlán, 1984).

\section{Ejemplo 8}

En este ejemplo se ha vulgarizado la significación original de la teoría física, a través de las múltiples connotaciones del término información. Un profesor de física, en una clase de relatividad especial, dice:

-Toda la información se propaga con [la] velocidad [de la luz] c (...); es el comportamiento ondulatorio.

\section{Ejemplo 9}

Este ejemplo es un caso en el que se utiliza un término científico en una clase de física, pero remitiéndolo a una supuesta significación desde otra disciplina, con el resultado de que se desnaturalizan y empobrecen ambas perspectivas. El profesor comenta:

-Picasso es relativista, porque, según desde dónde lo mires, se ve distinto.

\section{Ejemplo 10}

Otro profesor de física explica, intentando caracterizar los conductores en ausencia de corriente eléctrica:

-Los electrones se quedan quietitos.
La sustitución patológica presente en estas afirmaciones (categoría $c$ ) se corresponde con la idea de «bajar la dificultad de los contenidos» a la altura del alumno con el supuesto fin de ayudar a su comprensión conceptual. Pero, en realidad, se pierde por el camino la denotación científica original, desvirtuando los conceptos al reducir su nivel de abstracción.

\section{La culpa es de los otros}

El saber aparece como el instrumento por excelencia a la hora de legitimar las relaciones de poder (Fernández Enguita, 1991), pero no ya desde la asociación tradicional entre saber/experiencia y autoridad, sino a través del discurso, que es frecuentemente irónico, y en el cual el alumno lleva las de perder.

En los siguientes ejemplos, el lenguaje, lejos de reforzar actitudes positivas de los alumnos, se transforma en un vehículo para la descalificación y la desvalorización actitudinal, en una triste negociación donde el «no entender» por parte del alumno es leído por el docente como un «no querer aprender». Asimismo, las situaciones «interesantes» para el alumno son descalificadas por el sistema de valores no explícito del docente.

\section{Ejemplo 11}

Un profesor de física caracteriza a sus alumnos lapidariamente:

[Ustedes] Son a-neuronados (sic) y maleducados.

\section{Ejemplo 12}

Otra profesora le dice a un alumno que logra percibir con esfuerzo una relación sustantiva entre conceptos, estando de pie frente a sus compañeros:

- ¡A buena hora lo descubre, señor!

\section{Ejemplo 13}

Durante una clase acerca de los reptiles, un alumno pregunta si pueden «abrir» una víbora o un sapo. El profesor, sin ofrecer un ámbito donde dialogar sobre «ética científica», lo descalifica:

\section{-Ustedes no se merecen aún la muerte de ningún animal.}

En todos los ejemplos de este apartado se ha transferido el problema de la fractura comunicativa a planos que exceden lo estrictamente didáctico.

En conversaciones informales con algunos docentes hemos podido detectar que, en aquéllos que manifiestan un bajo sentimiento de pertenencia institucional, se encuentra alta resistencia al cambio de sus prácticas, aun cuando toman conciencia del fracaso reiterado de sus alumnos. Esta situación se extiende a quienes no son profesionales de la enseñanza, sino que han sido «expulsados-a-la-carrera-docente» por razones laborales de fuerza mayor. 
Los mecanismos para legitimar esta resistencia son variados. Puede verse que la injerencia alta de la institución en los contenidos funciona como «coartada», en excusas tales como:

-[No puedo detenerme en indagar qué entiende cada alumno,] tengo que cumplir con los ejercicios de la guía del Departamento.

En otros casos, expresiones tales como:

-Hago como que los alumnos no existen, porque si no, me enfermo.

Eso demuestra una completa negación del rol del educador, aun siendo concientes de sus deficiencias como facilitadores del aprendizaje.

La voluntad para el cambio incluye en sí misma una componente mediacional (de reflexión sobre el propio discurso) al estar relacionada con pensar lo impensable y desconstruir la propia práctica pedagógica alienada (Carrizales, 1993). Si el docente no se siente analizador de su rol de comunicador de contenidos buscando posibles disfunciones, no puede situarse en una posición que favorezca la renovación del discurso tradicional, y sólo se esgrimen mecanismos «defensivos».

Muchos de los docentes observados ni siquiera percibieron las disfunciones lingüísticas en su discurso, ni la forma en la que los alumnos asumen pasivamente la incomprensión de la situación comunicativa.

\section{CONCLUSIONES}

Una vez realizadas las observaciones y analizados los registros desde el marco teórico abordado, llegamos a tres conclusiones principales:

\section{El docente no se percibe a sí mismo como comuni- cador}

La conclusión más fuerte que puede extraerse de las observaciones es que casi en ningún caso el docente reflexiona acerca de su rol de comunicador de la estructura lingüística de su asignatura (conclusión similar a la de Borsese [1994], pero retomada desde otra perspectiva). A pesar de que se considera a sí mismo «enseñante» de los contenidos de una disciplina, desconoce que el lenguaje natural está funcionando como el mediador de la práctica y como el sustento de la construcción del sublenguaje científico (Michinel y D'Alessandro, 1994). Este desconocimiento lo lleva a restar importancia a las diferencias profundas entre el lenguaje del sentido común y el científico, diferencias que cumplen un papel crucial a la hora de facilitar u obturar los buenos aprendizajes (Pozo et al., 1991).

\section{La sutileza de los mecanismos del vaciamiento del discurso escolar}

Otra conclusión importante es la sutileza de los mecanismos del vaciamiento discursivo escolar. En la mayoría de los casos, es necesario un experto en contenidos situado fuera de la situación comunicativa y con fuerte formación metadisciplinar (el rol que cumplieron en mayor o menor medida los observadores) para detectar la disfunción. Ésta es una conclusión particularmente interesante, porque el observador usual de situaciones de clase (directivo, inspector) realiza, generalmente, un análisis desde lineamientos más superficiales (si el docente tiene buena voz, si mantiene a sus estudiantes atentos, si los hace participar, si su clase tiene principio y cierre, etc.), pero su desconocimiento del área disciplinar específica le dificulta captar la esencia del vaciamiento discursivo.

\section{El practicante (futuro profesor) como observador pertinente}

Cabe contextualizar nuevamente nuestro trabajo a la luz del planteo inicial de estudio de las prácticas pedagógicas en el marco de la formación docente, a través del muy difundido método de «observaciones». Interesa destacar el valor de la existencia de un espacio de reflexión acerca de la comunicación en el aula, para transformar al profesional docente en un investigador de su propia práctica.

Este espacio, al ser instaurado en la formación docente anterior a la práctica, repercutirá en la lectura que el docente pueda hacer de los problemas de comunicación en el aula que se presentarán en su desempeño. Por un lado, situará al lenguaje como objeto de estudio y de reflexión, otorgánole un rol importante en el análisis de las dificultades de enseñanza y aprendizaje. Por otro lado, problematizará las miradas ingenuas en las que las dificultades son atribuidas unilateralmente a los alumnos. Además, permitirá construir soluciones que atiendan a la mejora de la eficacia comunicativa, condición necesaria para la genuina compartición de significaciones científicas.

Todas estas reflexiones, sin embargo, están condicionadas a la capacidad del profesor en formación de considerarse a sí mismo como comunicador («usuario» del lenguaje), y explorar su discurso buscando las fracturas. Creemos que esta capacidad puede ser alentada durante la formación, y el espacio de «observaciones» es una instancia muy fuerte para vivenciar estas reflexiones en la acción.

Por otra parte, la presencia de practicantes como observadores especialistas tanto en temas del área disciplinar como del área pedagógica, lejos de situar a los docentes observados en un aula de paredes de vidrio, debería propender al enriquecimiento mutuo de:

-los observadores, que recogen la experiencia de docentes en actividad, encontrándose con su desempeño en el aula «real», y se nutren así de ejemplos de «uso eficaz» del lenguaje;

-los observados, a través de informes o comentarios («retroalimentación») que pongan en relieve la percepción de estereotipos y vicios comunicativos. 


\section{REFERENCIAS BIBLIOGRÁFICAS}

ANDER-EGG, E. (1980). Técnicas de investigación social. Buenos Aires: El Cid Editor.

AUSUBEL, D., NOVAK, J. y HANESIAN, H. (1983). Psicología educacional: un punto de vista cognoscitivo. Trillas: México.

BONÁN, L. y ADÚRIZ BRAVO, A. (1995). «Análisis de prácticas pedagógicas a través de la observación de clases en el contexto de la formación docente», Informe de Investigación, mimeo. Buenos Aires: CEFIEC, FCEyN, UBA.

BORSESE, A. (1994). Il problema della comunicazione linguistica a scuola: il linguaggio scientifico e chimico in particolare. Enseñanza de las Ciencias, 12(3), pp. 333-337.

CAÑAL,P.y PORLÁN, R. (1987). Una experiencia de aprendizaje por investigación directa del medio en la formación de maestros. Revista de Educación, 284, p. 273.

CARRIZALES, C. (1993). Alienación y cambio en la práctica docente, en Alliaud, A. et al. (comp.). Maestros: formación, práctica y transformación escolar. Buenos Aires: Miño y Dávila.

CHEVALLARD, Y.(1997). La transposición didáctica: Buenos Aires: Aique.

CONTRERAS, J. (1990). Enseñanza, currículum y profesorado. Introducción crítica a la didáctica. Madrid: Akal.

EASLEY Jr, J.A. (1982). Naturalistic case studies exploring social-cognitive mechanisms, and some methodological issues in research of problems of teachers. Journal of Research in Science Teaching, 19(3), pp. 191-203.

EDWARDS, D. y MERCER, N. (1988). El conocimiento compartido. Buenos Aires: Paidós.

ENTEL, A. (1988). Escuela y conocimiento. Buenos Aires: Miño y Dávila.

ERICKSON, G. y TIBERGHIEN, A. (1989). Calor y temperatura, en Driver, R. Guesne, E. y Tiberghien, A. (comp.). Ideas científicas en la infancia y la adolescencia. Madrid: Morata.
FERNÁNDEZ ENGUITA, M. (1991). La escuela a examen Madrid: Eudema Actualidad.

FRIGERIO, G. (comp.) (1991). Curriculum presente, ciencia ausente. Buenos Aires: Miño y Dávila.

GAETA, R. et al. (1996). Modelos de explicación científica. Problemas epistemológicos de las ciencias naturales y sociales Buenos Aires: Eudeba.

GALAGOVSKY, L.R. (1993). Hacia un nuevo rol docente. Una propuesta diferente para el trabajo en el aula. Buenos Aires: Troquel.

GALAGOVSKY,L.R. (1996). Redes conceptuales: aprendizaje, comunicación y memoria. Buenos Aires: Lugar Editorial.

GIMENO SACRISTÁN, J. y PÉREZ GÓMEZ, A. (1985). La enseñanza: su teoría y su práctica. Madrid: Akal.

LAHORE, A. (1993). Lenguaje literal y connotado en la enseñanza de las ciencias. Enseñanza de las Ciencias, 11(1), pp. 59-62.

MERZYN, G. (1987). The language of school science. International Journal of Science Education, 4, p. 483.

MICHINEL, J.L. y D'ALESSANDRO MARTÍNEZ, A. (1994). El concepto de energía en los libros de textos: de las concepciones previas a la propuesta de un nuevo sublenguaje. Enseñanza de las Ciencias, 12(3), pp. 369-380.

POZO, J.A., SANZ, J.A., GÓMEZ CRESPO, M.A. y LIMÓN, M. (1991). Las ideas de los alumnos sobre la ciencia, una interpretación desde la psicología cognitiva, Enseñanza de las Ciencias, 9(1), pp. 83-94.

SÉRÉ, M.G. (1989). El estado gaseoso, en Driver, R. Guesne, E. y Tiberghien, A., (comp.). Ideas científicas en la infancia y la adolescencia. Madrid: Morata.

VYGOTSKY, L.S. (1993). Pensamiento y lenguaje. Buenos Aires: Ediciones Fausto.

[Artículo recibido en octubre de 1996 y aceptado en junio de 1997.] 